\title{
ReIMAGINE: a prostate cancer research consortium with added value through its patient and public involvement and engagement
}

\author{
S. Green ${ }^{1}$, S. Tuck ${ }^{2 \dagger}$, J. Long ${ }^{2 \dagger}$, T. Green ${ }^{2 \dagger}$, A. Green ${ }^{2 \dagger}$, P. Ellis ${ }^{2 \dagger}$, A. Haire ${ }^{1}$, C. Moss ${ }^{1}$, F. Cahill ${ }^{1}$, N. McCartan ${ }^{3,4}$, \\ L. Brown', A. Santaolalla', T. Marsden ${ }^{3,4}$, M. Rodriquez Justo ${ }^{3,4}$, J. Hadley ${ }^{3,4}$, S. Punwani ${ }^{6}$, G. Attard ${ }^{3}$, H. Ahmed ${ }^{7}$, \\ C. M. Moore ${ }^{3,4}$, M. Emberton ${ }^{3,4}$ and M. Van Hemelrijck ${ }^{1 *}$ (1)
}

\begin{abstract}
Background: ReIMAGINE aims to improve the current prostate specific antigen (PSA)/biopsy risk stratification for prostate cancer (PCa) and develop a new image-based method (with biomarkers) for diagnosing high/low risk PCa in men. RelMAGINE's varied patient and public involvement (PPI) and engagement (PE) strategy maximises the impact of its scientific output by informing and shaping the different stages of research.

Aims: Through including the voice of patients and the public, the ReIMAGINE Consortium aims to translate these different perspectives into the design and implementation process. This will improve the overall quality of the research by:

- reflecting the needs and priorities of patients and the public, ensuring methods and procedures are feasible and appropriate

- ensuring information is relevant and accessible to those being recruited to the study

- identifying dissemination channels relevant to patients/the public and developing outputs that are accessible to a lay audience

With support from our patient/user groups, the ReIMAGINE Consortium aims to improve our ability to derive prognostic information and allocate men to the most appropriate and effective therapies, using a novel image-based risk stratification with investigation of non-imaging biomarkers.
\end{abstract}

Findings: We have been working with patients and the public from initiation of the project to ensure that the research is relevant to men and their families. Our PPI Sub-Committee, led by a PCa patient, has been involved in our

\footnotetext{
*Correspondence: mieke.vanhemelrijck@kcl.ac.uk

'`. Tuck, J. Long, T. Green, A. Greene, and P. Ellis: Patient author.

${ }^{1}$ Translational Oncology and Urology Research (TOUR), School of Cancer

and Pharmaceutical Sciences, King's College London, London, UK

Full list of author information is available at the end of the article

This paper is dedicated to the memory of Alan Greene, our friend and

colleague who sadly passed away before this paper was published.
}

(c) The Author(s) 2021. Open Access This article is licensed under a Creative Commons Attribution 4.0 International License, which permits use, sharing, adaptation, distribution and reproduction in any medium or format, as long as you give appropriate credit to the original author(s) and the source, provide a link to the Creative Commons licence, and indicate if changes were made. The images or other third party material in this article are included in the article's Creative Commons licence, unless indicated otherwise in a credit line to the material. If material is not included in the article's Creative Commons licence and your intended use is not permitted by statutory regulation or exceeds the permitted use, you will need to obtain permission directly from the copyright holder. To view a copy of this licence, visit http://creativecommons.org/licenses/by/4.0/. The Creative Commons Public Domain Dedication waiver (http://creativeco mmons.org/publicdomain/zero/1.0/) applies to the data made available in this article, unless otherwise stated in a credit line to the data. 
dissemination strategy, outreach activities, and study design recommendations. For example, the sub-committee have developed a variety of informative videos relevant and accessible to those being recruited, and organised multiple online research engagement events that are accessible to a lay audience. As quoted by one of the study participants, "the more we present the benefits and opportunities to patients and the public, the more research commitment we obtain, and the sooner critical clinical questions such as PCa diagnostics will be addressed".

Keywords: Patient and public involvement, Patient and public engagement, Cancer, Prostate cancer, Study design

\section{Plain English Summary}

One in eight men will be diagnosed with prostate cancer (PCa). Most will not die of it, but our ability to identify those men whose cancer poses the greatest threat to life has, thus far, been poor. Some men are diagnosed with small cancers which will never cause them a problem, some will have treatment which is unnecessary, others will have their cancers missed, and others will be misclassified as either having low risk cancer and will therefore miss out on the appropriate treatment, or told their cancer is high risk and have unnecessary treatment. Nowhere else in modern medicine are these errors of over-diagnosis, over-treatment, missed-diagnoses, and poor risk-stratification more common.

The ReIMAGINE Consortium has been developed to undertake discoveries that will correct these four key errors in the PCa diagnostic pathway. We will investigate how to best identify which men have, or will develop, aggressive prostate cancer using imaging combined with advanced biomarker analyses of blood and urine (i.e., OMICs technologies such as whole genome sequencing, targeted sequencing (e.g.:=, methylation). We will achieve this by building on established partnerships between patients, advocacy organisations, clinicians, imaging experts, molecular biologists, methodologists, and a broad range of industrial partners.

The Patient and Public Involvement (PPI) sub-committee is an integral part of the study workflow, contributing to study design and recruitment, results analysis, and dissemination. The committee, led by a funded PPI co-ordinator and a patient chair, have given invaluable insight into the study modifications due to COVID-19 restrictions.

\section{Background}

ReIMAGINE, a consortium funded by the Medical Research Council (MRC) and Cancer Research UK (CRUK), was established in 2018 to further refine the use of magnetic resonance imaging (MRI), in combination with diagnostic molecular markers, as a tool for precise baseline risk stratification of men being assessed for prostate cancer $(\mathrm{PCa})$. This work is needed as, unfortunately, for men with apparently low risk $\mathrm{PCa}$, it is not yet possible to distinguish those tumours that will remain indolent and those that will develop into high risk or advanced disease [1].

ReIMAGINE aims to recruit 1,000 men referred to secondary care with a suspicion of PCa or men who are undergoing further tests for PCa staging into a prospective cohort study, with detailed assessment of baseline characteristics (i.e., clinic-demographics as well as (liquid) biopsies and imaging) and life-long follow-up (study A). In addition, Re-IMAGINE will run a pilot screening study aiming to evaluate MRI as a screening tool in 300 men (study B).

We have been working with patients and their families from initiation of the project and this has recently also led to recruitment completion for study B despite COVID19 limitations (see below). Here, we describe how active patient and public involvement (working in partnership with patients and members of the public to plan, manage, design, and carry out research) and engagement (how information and knowledge about research is provided and disseminated) with patients and the public from inception leads to impactful evidence-based clinical research outputs for the ReIMAGINE Consortium and the studies within the project. It is important to point out that "PPI takes various forms, from involvement of public contributors in priority setting, representation on committees, and as reviewers" [2]. The latter 2 forms have been invaluable to our ongoing research processes.

\section{ReIMAGINE patient and public involvement (PPI) structure and processes}

From the outset of this project, the ReIMAGINE Consortium operated on the premise of 'no decision about the patient, without the patient.' The grant application was developed with both patient and public involvement $[3,4]$, and a patient was part of the team who were interviewed at the final stage of the application. During the grant writing/planning phase, we used a three-stage approach (i.e., information session, discussion groups, and feedback presentation), whereby we interacted 
with patients, their families, as well as men from the general population to develop an active partnership between patients and researchers $[5,6]$. This collaboration between researchers and patients has identified and developed Re-IMAGINE in such a way that the output will be relevant and beneficial to patients and their needs.

Once funded, we appointed a PPI Coordinator to work within the PPI\&E workstream. We began with a series of discussion groups, whereby patients and their family members, as well as men without PCa (i.e. the general public) provided insight into participant preferences with respect to design and management, data collection and analysis, and dissemination of findings. We had several meetings and ensured that the ethnic diversity of London was represented. The meetings were scheduled alternatively during the daytime and in the evening, to allow a wide range of patients and members of the public to engage. Following these discussion groups, we also presented the project for feedback to the South East London Consumer Research Panel and at the Guy's Cancer Survivors Day (June 2017) for further feedback.

\section{Recommendations from PPI}

The discussions confirmed the research need for less invasive diagnostic strategies for prostate cancer and generated study design recommendations. For instance, the participants of the discussion groups recommended us to speak with general practitioners (GPs) to ensure we had an efficient recruitment strategy for study B. A discussion group with 10 London-based GPs was therefore held to identify the best method for identifying communitybased men eligible for study B. The GPs were asked about uptake and participation rates, the best method for contacting eligible participants, the possibility of collecting semen in addition to blood (something we then chose not to do), how to best inform the GP practice about the participants' MRI results (especially when there is a possible PCa diagnosis), IT solutions, and reimbursements. All feedback was accounted for when submitting approval for the screening study. Over 10 GP Surgeries based in North, South and East London received approval to participate in this study.

In the context of our PPI and engagement strategy, we discussed project reporting, patient representation in the steering committee, the need for a PPI Sub-Committee, and a dissemination strategy. For the latter, we involved ecancer, one of the leading oncology communication charities. We were also encouraged to reach out to Prostate Cancer UK and Movember, two of the UK's leading PCa patient support charities, which helped us to identify the chair for our PPI Sub-Committee. Mr Tuck was a Movember ambassador at the time and also leads a PCa patient support group in Oxfordshire. Hence, our discussion groups also provided an opportunity to build relationships with patient communities and involve individual patients going forward, as recommended by Doria et al. [7].

\section{Establishing the PPI sub-committee}

Recruitment for PPI can often be challenging, however with the support of colleagues, such as, Prostate Cancer Clinical Nurse Specialist and Clinical Research Team we were able to find patients for the sub-committee. Such colleagues are a useful resource as they work directly with patients and have established relationships and can therefore suggest which patients might be willing to be involved in research. In addition, we also had the support of charities such as PCUK and Movember. Forming alliances with different teams/bodies has assisted us with our committee numbers and representation, working with our brief which specifies people of diverse backgrounds, in particular, men from the black community, given they are at a significantly increased risk of prostate cancer.

The PPI Sub-Committee, chaired by Mr Tuck, meets consistently every three months to ensure that the needs and priorities of participants are practical and suitable as well as to ensure information is relevant and accessible to those being recruited and the public. The committee consists of two white men and one black man with prostate cancer between the ages of 40-80 years old, with varying educational qualifications. In addition, one black woman (age 43) whose father died of prostate cancer, the ReIMAGINE PPI Coordinator, and a research academic sit on the committee. The diversity of the group has permitted a greater range of perspectives from different age groups and communities to help shape the research. The Chair also attends fortnightly project meetings with the wider team and feeds back to the sub-committee accordingly and vice versa.

\section{The PPI sub-committee roles}

Following the establishment of a terms of reference, outlining the aims and objectives of the group, the committee have reviewed all patient facing documents (e.g. patient information sheet, consent form, invitation letter), developed the patient area on the ReIMAGINE website, advising on design, layout and content. They created the storyboard and scripts for the patient information videos on the website by working closely with the PPI Coordinator and ecancer and engaged in the production process of the six-monthly newsletter through feedback on both content and design. The Chair also assists with editing the final draft of the newsletter. 


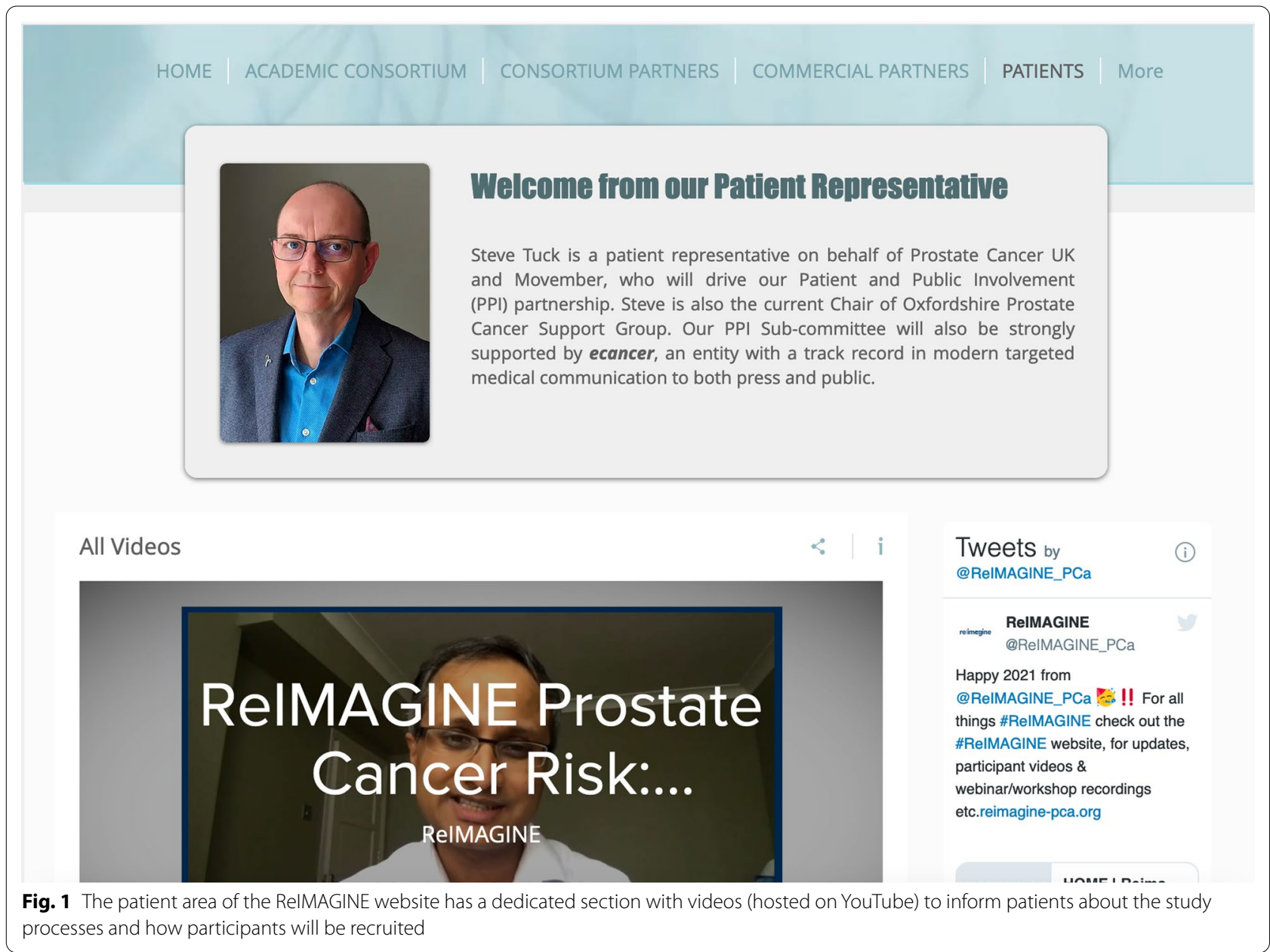

\section{Prostate cancer specialist PPI sub- group}

Additionally, through the active involvement and engagement of our PPI Sub-Committee, we have launched a specialist PCa Research Group for Black, Asian and Minority Ethnic communities, following the Black Lives Matter Movement in 2020. This specialist group provides a platform for a greater range of perspectives and allows researchers to access and foster dialogue between this under-represented population of patients and members of the public who wish to be involved with clinical research, in addition to providing an opportunity for their voices to be amplified through sharing their lived experiences - stimulating discussion and promoting greater diversity in research. We actively sought participation from men of diverse backgrounds and promoted our PPI group on Twitter, which has resulted in researchers from other institutions approaching us for support with their patient engagement activities. The ReIMAGINE PPI Coordinator was also selected to provide input for CRUK's Equality, Diversity and Inclusion Strategy.

\section{ReIMAGINE outreach and engagement}

The ReIMAGINE newsletter is written so that it is of interest to patients, the general public, healthcare professionals, and members of the ReIMAGINE Consortium. This patient-friendly update, as well as every other component of the PPI Sub-Committee's dissemination strategy, is co-produced with ecancer who also supports the development of the videos, leaflets, flyers, banners, and invitations. The ReIMAGINE website has also been designed to be a source of information for researchers, healthcare professionals, and industry, as well as patients and the general public. The patient area has a dedicated section with videos (hosted on YouTube) to inform patients about the study processes and how participants will be recruited (Fig. 1). Members of the PPI Sub-Committee not only informed the content of these, but also participated as actors in the videos.

In addition, the PPI Sub-Committee engages in outreach activities throughout the UK to create awareness about PCa and the ReIMAGINE Consortium. Members 


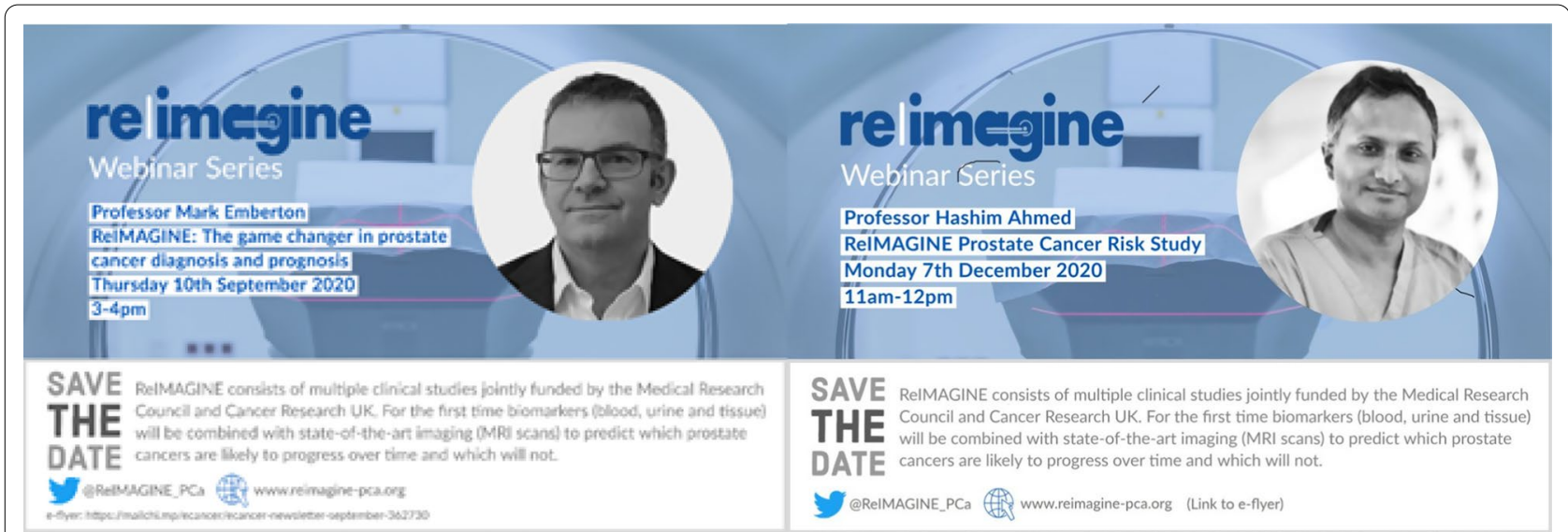

Fig. 2 To further engage with the prostate cancer community outside of London, we have been organising free webinar series which were advertised widely with the help of CRUK

advised on appropriate activities and what aspects of the study might be of interest to the public. Some members assisted with manning the stands on the respective days. For example, we have engaged in the International Clinical Trials Day at Guy's Hospital (2019), an annual event where researchers have the opportunity to engage with patients, public and staff about their project; the Cancer Survivors' Day at Guy's Cancer Centre (2018 and 2019), a showcase of fashion, music and research via seminars and exhibits; and the Open Research Day of University College Hospital London (2018), a celebration of research; as well as participated in outreach activities of the Jamaica UK Diaspora in Birmingham. We also advertised a ReIMAGINE banner on the conference app of the 2019 Conference of the National Cancer Research Institute. To further engage with the prostate cancer community outside of London, we worked in partnership with charities and patient groups nationwide to promote our free webinar series (Fig. 2), which was moderated by our PPI Sub-committee Chair. In addition, each speaker's presentation was reviewed by the Chair in order to ensure that the content was relevant and accessible to a lay audience. The PPI Sub-committee also produced a research article in one of the newsletters from Tackle - an umbrella organisation for $>90 \mathrm{PCa}$ support groups in the UK. The PPI sub-committee members have played a crucial role in identifying different dissemination channels that are relevant to patients/the public and developing outputs that are accessible to a lay audience.

Twitter (@reimagine_pca) has been an invaluable platform for our research engagement as it allows us to participate in many other relevant PPI activities online: e.g. Urology Awareness month, World Cancer Research Day, Urology week, London Global Cancer Week, Prostate
Cancer Awareness month, Black History Month, and International Men's Day. The latter was also the day on which we had our first online research engagement event (19th November 2020) to inform the wider public about ReIMAGINE. This event was an evening of updates, interactive sessions and workshops with ReIMAGINE team members Prof Mark Emberton, Prof Hashim Ahmed, Prof Caroline Moore, and Prof Gerhardt Attard. The event also included an interview conducted by the PPI Sub-Committee chair with a participant. The PPI Sub-Committee was heavily involved in the organisation of this event, which was well received by the attendees. One prostate cancer patient representative who attended the event emailed to say that; "The webinar was 'gold standard' patient and public engagement. The particularly positive elements were: The session felt as if it had been co-produced with the PPI lead.

- Always positive to share results with patients and the public

- The agenda was well thought through

- The content was generally understandable to a lay audience and presentations were clear

- The men who'd participated in the study were thanked

"Your time commitment to this, shows your commitment to sharing research with lay audiences. Thank you. For me the more we present the benefits and opportunities research brings to patients and the public, the more we get commitment for them to be involved in future trials, the sooner we get answers to the critical research." 


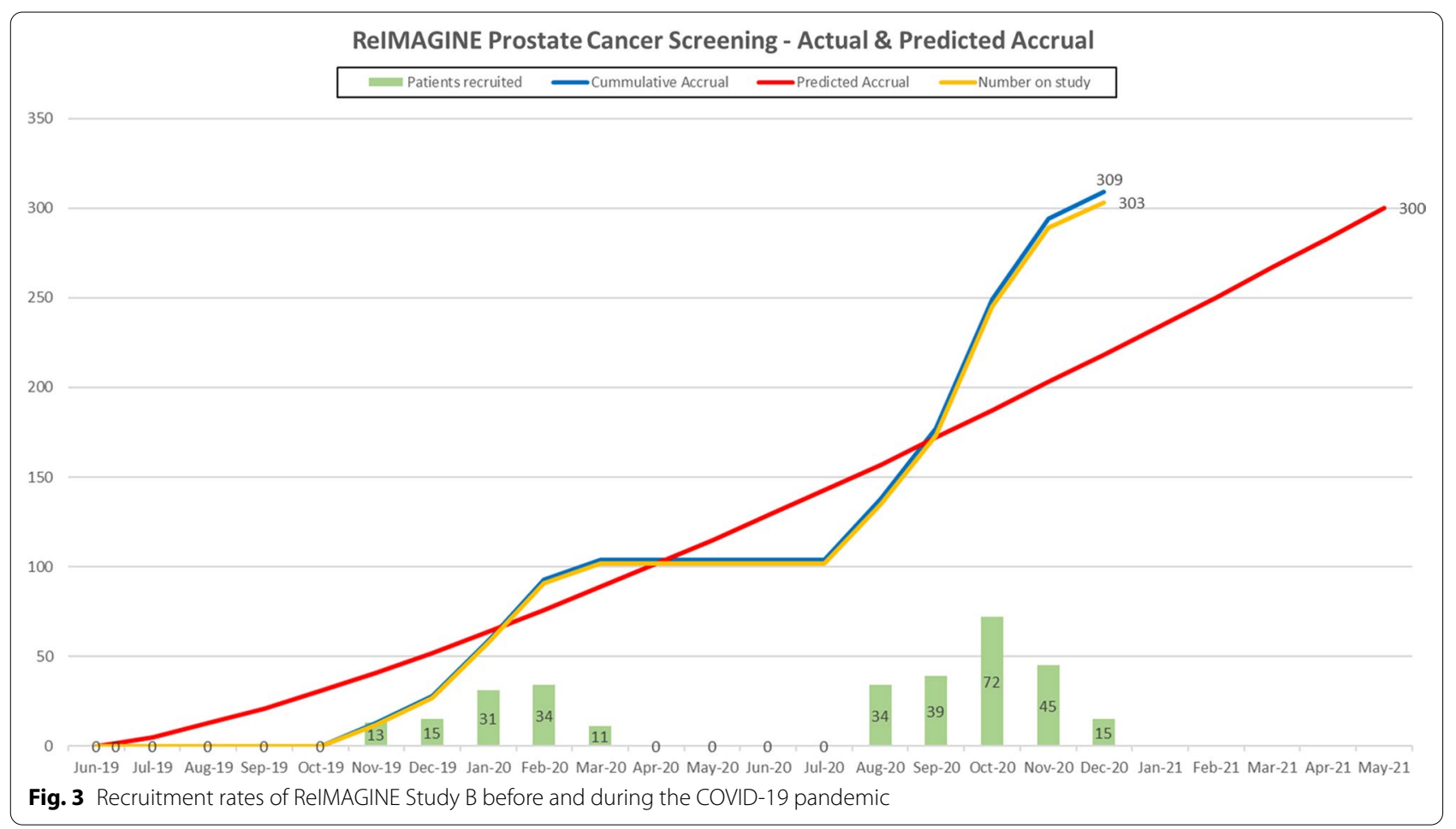

\section{An example of our PPI impact: overcoming the challenges of COVID}

Importantly, we would also like to report how our PPI strategy has helped ReIMAGINE overcome some of the research limitations due to COVID-19. In March 2020, NHS trusts and research sponsors implemented a variety of policies based on the interpretation of nationwide government sanctioned guidelines. Introduction of these policies resulted in the closure of all non-COVID related research from midway through that month. After a period of time NHS trusts and study sponsors developed processes of special exemption to reopen active recruitment. The Trial management groups worked closely with the PPI Sub-committee to ensure the modifications being implemented reflected the needs and priorities of patients and the public. Consequently, we developed a roadmap for reopening of the two studies. The committee members raised ideas that were not considered by the consortium, for instance, inclusion of COVID-19 specific and hygiene related information in the PIS/Invitation letter such as;

- how the MRI scanner is decontaminated

- no physical contact; no examination, no doctor present

- healthcare staff will have adequate PPE

- special provisions for BAME men, and men with comorbidities
In addition, we developed COVID-19 Safeguarding videos together with the PPI Sub-Committee and ecancer for both study A and study B, in which men clearly explained how we mitigated potential COVID-19 risks for those participating in ReIMAGINE. The PPI subcommittee also suggested study modifications to reassure men of the safety of participation, e.g. paid taxi travel in study B. This advice was not only useful in terms of its acceptability to the potential participants, but provided reassurance to the study sponsors, NHS trusts and National Research Ethics committees that study modifications were guided by patient opinion.

Recruitment for Study A is currently limited due to the ongoing COVID-surge in the UK, but Study B managed to recover impressive recruitment rates following the first COVID wave and completed recruitment in December 2020 (Fig. 3). Input from the PPI sub-committee has been invaluable, ensuring that appropriate modifications are in place to maximise patient, researcher and NHS safety. Thus increasing the number of potential patients and the uptake rate.

\section{Conclusions}

The PPI strategy of ReIMAGINE ensures direct patient impact. The consortium has incorporated structures and funding for inclusion and engagement of the patient and public voice in the study design, monitoring and ongoing processes, resulting in a more effective and improved 
research process. The development of robust PPIE structures and processes that are embedded in the whole research process ensures that encountered challenges can be overcome demonstrated by the overcoming of recruitment challenges during the COVID-19 pandemic. The appointment of a funded PPI co-ordinator and a patient chair of the PPI sub-committee has led to further work outside the study remit, particularly in the establishment of a specialist PPI committee for PCa for people of diverse ethnic backgrounds.

\section{Abbreviations}

BAME: Black, Asian and Minority Ethnic; GP: General practitioner; MRI: Magnetic resonance imaging; PCa: Prostate cancer; PIS: Patient information sheet PPI: Patient and public involvement.

\section{Acknowledgements}

Caroline M Moore receives funding from Prostate Cancer UK, Movember, the Medical Research Council, Cancer Research UK and the NIHR. Mark Emberton receives research support from the United Kingdom's National Institute of Health Research (NIHR) UCLH/UCL Biomedical Research Centre. Shonit Punwani receives research support from the United Kingdom's National Institute of Health Research (NIHR) UCLH/UCL Biomedical Research Centre. Mieke Van Hemelrijck receives funding from Cancer Research UK. Katharina Beyer kindly supported the revisions of the manuscript. We would also like to thank all the members of the ReIMAGINE Consortium for their time and support: Coolen T Tuck S, Corbett G, Wingate A, Akbar F, Pervez H, Thakali S, Henderson A, Tekin D, Brembilla G, Gong F, Giganti F, Rawlins F, Tam H, Bholastewart H, Keskin S, Bertoncelli M, Maynard W, Bevan C, Boutros P, Feber A, Whitaker H, Freeman A, Domany E, Mason M, Padhani A, Aboagye E, Kaplan R, Parker C, Parker P, Sidhu H, Kopcke D, Isaac E, Beeston T, Clement J, Soteriou K.

\section{Authors' contributions}

Data collection: SG, ST, JL, FC, TG, AG, PE, AH, CM, NM, AS, CM, ME, MVH, TM. Engagement in PPI activities: $S G, S T, J L, T G, F C, A G, P E, A H, C M, N M, L B, T C, A S$, $T M, S P, A G, C M, E M, M V H, K B$. First draft of manuscript: SG, MVH. Critical revision of manuscript: SG, ST, JL, TG, AG, PE, AH, CM, FC, NM, LB, AS, TM, JS, JD, SP, GA $\mathrm{HA}, \mathrm{CM}, \mathrm{ME}, \mathrm{MVH}, \mathrm{KB}$. All authors read and approved the final manuscript.

\section{Funding}

ReIMAGINE Screening is funded by The Medical Research Council, U.K. (MRC), grant number MR/R014043/1 and Cancer Research U.K. (CRUK).

\section{Availability of data and materials}

Not applicable.

\section{Declarations}

Ethics approval and consent to participate

ReIMAGINE Prostate Cancer Screening has regulatory approval from the Regional Ethics Committee London - Stanmore (reference 19/LO/1129).

\section{Consent for publication}

Not applicable.

\section{Competing interests}

Caroline M Moore receives fees for HIFU proctoring from SonaCare. She has received speaker fees from Astellas, and Jannsen. She carries out research into photodyanamic therapy supported by Spectracure. Mark Emberton serves as a consultant/educator/trainer to Sonacare Inc., Exact Imaging, Angiodynamics Inc and Profound Medical. Acknowledgements.

\section{Author details}

${ }^{1}$ Translational Oncology and Urology Research (TOUR), School of Cancer and Pharmaceutical Sciences, King's College London, London, UK. ReIMAGINE Consortium Patient Representative, London, UK. ${ }^{3}$ UCL Division of Surgical and Interventional Sciences, University College London, London, UK. ${ }^{4}$ Department of Urology, University College London Hospitals NHS Foundation Trust, London, UK. ${ }^{5} \mathrm{MRC}$ Clinical Trials Unit, University College London, London, UK. ${ }^{6}$ Centre for Medical Imaging, University College London, London, UK. ${ }^{7}$ Imperial College, London, UK.

Received: 29 January 2021 Accepted: 27 October 2021

Published online: 17 November 2021

\section{References}

1. Carter HB. Prostate-specific antigen (PSA) screening for prostate cancer: revisiting the evidence. JAMA. 2018;319(18):1866-8.

2. Research NIfH. Glossary of Terms. https://www.nihr.ac.uk/about-us/gloss ary.htm?letter=P\&postcategory $=-1$.

3. Gooberman-Hill R, Burston A, Clark E, Johnson E, Nolan S, Wells V, et al. Involving patients in research: considering good practice. Musculoskeletal Care. 2013;11(4):187-90.

4. McCarron TL, Noseworthy T, Moffat K, Wilkinson G, Zelinsky S, White $D$, et al. A co-designed framework to support and sustain patient and family engagement in health-care decision making. Health Expect. 2020;23(4):825-36.

5. Bagley HJ, Short H, Harman NL, Hickey HR, Gamble CL, Woolfall K, et al. A patient and public involvement (PPI) toolkit for meaningful and flexible involvement in clinical trials - a work in progress. Res Involv Engagem. 2016;2:15.

6. Society BG. Patient and public involvement in research 2018. https:// www.bgs.org.uk/resources/patient-and-public-involvement-in-resea rch\#: : text=\%20Patient\%20and\%20public\%20involvement\%20in\%20res earch\%20,about\%20the\%20challenges\%20as\%20well\%20as...\%20More\% 20.

7. Doria N, Condran B, Boulos L, Curtis Maillet DG, Dowling L, Levy A. Sharpening the focus: differentiating between focus groups for patient engagement vs qualitative research. Res Involv Engagem. 2018;4:19.

\section{Publisher's Note}

Springer Nature remains neutral with regard to jurisdictional claims in published maps and institutional affiliations. 